OPEN

SUBJECT AREAS:

ELECTRONIC PROPERTIES

AND MATERIALS

NANOSCALE MATERIALS

ELECTROCHEMISTRY

BATTERIES

Received

24 April 2013

Accepted

15 July 2013

Published

30 July 2013

Correspondence and requests for materials should be addressed to

X.H. (xhuang@ntu. edu.sg) or H.H.H. (ashhhng@ntu.edu.sg)

\section{Carbon inverse opal entrapped with electrode active nanoparticles as high-performance anode for lithium-ion batteries}

\author{
Xin Huang, Jing Chen, Ziyang Lu, Hong Yu, Qingyu Yan \& Huey Hoon Hng
}

School of Materials Science and Engineering, Nanyang Technological University, 50 Nanyang Avenue, Singapore 639798, Singapore.

Enhancing ion and electron transport kinetics together with improving cycle life are important issues to be considered when developing high-performance Li ion batteries. Here we demonstrate a three dimensional ordered macroporous conductive electrode concept by entrapping electrode active nanoparticles in an interpenetrating macroporous carbon inverse opal. The electrodes are featured with simultaneously enhanced ion and electron transport kinetics as well as geometrically constrained active nanoparticles. The electrode can deliver up to $94.17 \%$ of theoretical capacity over 1000 discharge/charge cycles at a current density of $2.0 \mathrm{~A} \mathrm{~g}^{-1}$, and exhibits good rate capability in the high current density range of 1.0-10.0 $\mathrm{A} \mathrm{g}^{-1}$. We hope that our findings will help pave the way for tailored design of many other sophisticated electrode materials in electrochemistry.

igh performance Li ion batteries (LIBs) are required to deliver high capacity and cycling stability at high current density ${ }^{1,2}$. To achieve these purposes, it is important to enhance the ion and electron transport kinetics as well as the stability of electrode active materials ${ }^{3-6}$. Nanotechnology together with carbon coating provides the possibility to fulfill these requirements ${ }^{7-10}$. As demonstrated, carbon-coated electrode active nanomaterials indeed exhibit improved rate capabilities and cycling stability due to the much smaller diffusion length and increased fracture strength compared to the bulk counterparts ${ }^{11-14}$. For these materials, fast transport kinetics have been achieved in one dimensional (1D) carbon nanotube- and two dimensional (2D) graphene nanosheet-based hybrid electrodes ${ }^{15-18}$. However in $1 \mathrm{D}$ and $2 \mathrm{D}$ electrodes, the fast electron transport is restricted at least in one dimension because of the structural anisotropy of the electrodes ${ }^{19-21}$. As a result, the ambipolar (ionic and electronic) diffusion will be strictly constrained along that dimension, which eventually slows down the transport kinetics in the entire battery. Such kinetics problems will become more severe, especially at high current densities.

Ideally, the electrode should consist of an ordered three-dimensional (3D) interconnected network, where a 3D continuous transport pathway allows fast ion and electron transport without constrained ambipolar diffusion in any dimension. Along this line, initial strides have been made to fabricate three-dimensional ordered macroporous (3DOM) electrodes by colloid templating, such as $\mathrm{Li}_{4} \mathrm{Ti}_{5} \mathrm{O}_{12}{ }^{22}, \mathrm{SnO}_{2}{ }^{20}$ and $\mathrm{TiO}_{2}{ }^{23}$. These electrodes indeed show promising potential in enhancing transport kinetics of batteries but only moderate rate performances and cycling stability are observed due to the fact that the macroporous structure mainly addresses ion transport rather than electron transport. In response to this challenge, that is, to afford the 3DOM electrodes better electronic conductivity, bicontinuous 3DOM electrode concept is proposed by depositing a layer of electrode active materials on pre-prepared 3DOM conductive inverse opal (e.g. carbon or nickel) or coating the 3DOM active materials with carbon materials $s^{5,24,25}$. Although these bicontinuous electrodes enable high initial capacity and remarkable rate capabilities, they are still unable to provide a long-term cycling stability. Due to the lack of geometrical constrains in these electrodes, the pulverized active materials induced by cracking inside the active layer will inevitably peel off, eventually leading to cycling instability. Structural stability of the active materials is a critical factor in achieving a long cycle life. This issue is very challenging, and has not been well addressed. Owing to the above obstacles, the synthesis of ideal electrodes with fast electron and ion transport, as well as high cycling 
stability would require new designing concepts that have a comprehensive consideration of all important issues in batteries, such as electrode architecture and configuration of active species.

Based on these considerations, we here report a new electrode concept that is based on the integration of a 3DOM carbon inverse opal with geometrically entrapped active nanoparticles (NPs). This concept is designed to simultaneously address the issues of transport kinetics and long-term cycling stability of batteries. Our proof-ofconcept studies are based on the fabrication of 3DOM carbon inverse opal entrapped with $\mathrm{SnO}_{2} \mathrm{NPs}\left(\mathrm{SnO}_{2} @ 3 \mathrm{DOM}\right)$ and $\mathrm{CoO} \mathrm{NPs}$ (CoO@3DOM). In this design, the 3DOM carbon inverse opal ensures an interconnected continuous conductive network for fast electron transport, and the nanometer thick walls provide short diffusion pathways for lithium ions to go through. More importantly, the ordered periodic structure of the electrode minimizes the anisotropy of ion and electron transport kinetics because of the intrinsically geometric symmetry of the electrode. The macropores of the inverse opal also afford an easy accessibility of Li ions, and the geometrical constrains of elastic carbon inverse opal can accommodate the volume changes of oxide nanoparticles upon $\mathrm{Li}^{+}$insertion/ extraction, completely circumventing the self-aggregation and peeling off of the active materials.

\section{Results}

Synthesis and characterization of the $\mathrm{SnO}_{2} @ 3 \mathrm{DOM}$ electrode. Colloidal crystal templating (CCT) is a versatile method to fabricate 3DOM architecture. Self-assembled silica, polystyrene (PS) and poly(methyl methacrylate) (PMMA) spheres are typically used as opal templates ${ }^{26-28}$. Precursors fill the void spaces in opal templates by capillary action, and when the templates are removed via heating or chemical leaching, an ordered 3DOM inverse opal structure can be revealed. Although the synthesis of 3DOM electrodes is conceptually simple, the key issue in achieving perfect inverse opal replicas is homogeneous infiltration of precursors in the void spaces of templates. The poor wetting of precursors to templates will cause the replicas to lose their interconnectivity feature ${ }^{29}$. Bearing this in mind, we choose self-assembled carboxylic polystyrene (COOH-PS) spheres with diameter of $240 \mathrm{~nm}$ as opal templates (Supplementary Fig. S1), and tannic acid-Sn ${ }^{4+}\left(\mathrm{TA}-\mathrm{Sn}^{4+}\right)$ complexes (Supplementary Fig. S2) as the precursors of carbon and $\mathrm{SnO}_{2}$. Since tannic acid has abundant phenolic hydroxyls, these complexes are able to interact with the carboxyl groups (COOH-) of the templates via intensive hydrophilic interactions ${ }^{30,31}$, thus promoting the homogeneous distribution of $\mathrm{TA}-\mathrm{Sn}^{4+}$ precursors on the templates. To prevent the swelling of colloidal crystal during infiltration, deionized water is used as the solvent instead of organic solvent. Other experimental parameters, such as calcination temperature and precursors dosage, have been optimized in order to achieve inverse opal with nanometer thickness walls. Vacuum annealing removes the COOH-PS opal template and allows the in-situ crystallization of $\mathrm{SnO}_{2}$. Simultaneously, tannic acid decomposes to carbon inverse opal due to its low decomposition temperature according to TGA analysis (Supplementary Fig. S3). A schematic diagram illustrating the synthesis route of $3 \mathrm{DOM}-\mathrm{SnO}_{2}$ is shown in Figure 1. The inverse replica typically inherits the face-centered cubic (fcc) symmetry of the COO-PS sphere array. The fcc macropore array is interconnected through windows where templating spheres are in direct contact. Note that the in-situ formed $\mathrm{SnO}_{2}$ NPs are spatially entrapped in the carbon inverse opal. The geometrical constrains of carbon inverse opal to the $\mathrm{SnO}_{2} \mathrm{NPs}$ not only ensure an interpenetrating continuous network for ion and electron transport, but also minimize the peel off of the active materials from electrode. In addition, the entire electrode is geometrically symmetric in three dimensions that will be beneficial for fast ion and electron transport in all directions. Our synthesis procedures are flexible to prepare other metal oxideentrapped 3DOM electrodes because of the versatile chelating ability of tannic acid towards transition-metal ions, and this is discussed in detail below.

Field emission scanning electron microscopy (FESEM) images in Figure 2a-c reveal that the $\mathrm{SnO}_{2} @ 3 \mathrm{DOM}$ exhibits highly ordered porous inverse opal structure, which has two types of periodic porosities, including large macropores with diameter of $220 \mathrm{~nm}$ formed by the COOH-PS spheres, and smaller mesopores with diameter of $40 \mathrm{~nm}$ formed by the contact points between the $\mathrm{COOH}$-PS spheres. The wall thickness of the inverse opal is around $20 \mathrm{~nm}$. The geometrical constrains of $\mathrm{SnO}_{2} \mathrm{NPs}$ in the carbon inverse opal are evident in the field emission transmission electron microscopy (FE-TEM) images. In Figure $2 \mathrm{~d}-\mathrm{f}$, the interconnected carbon inverse opal is orderly packed, and small $\mathrm{SnO}_{2}$ NPs are entrapped in the entire framework. The macroporous framework consists of very thin amorphous carbon wall, and the entrapped $\mathrm{SnO}_{2} \mathrm{NPs}$ with diameter of $4 \sim 10 \mathrm{~nm}$ are quite well dispersed (Figure $2 \mathrm{f}$ ). High resolution TEM (HR-TEM) image of the $\mathrm{SnO}_{2}$ NPs in Figure $2 \mathrm{~g}$ shows the lattice spacing of $2.3 \AA$ corresponding to the $\{111\}$ plane of tetragonal $\mathrm{SnO}_{2}$ (JCPDS No. 41-1445), and the polycrystalline nature of these NPs is confirmed by the selected area electron diffraction (SAED) (Figure 2h). The presence of tetragonal $\mathrm{SnO}_{2}$ in $\mathrm{SnO}_{2} @ 3$ DOM is also identified by X-ray diffraction (XRD) patterns analysis (Supplementary Fig. S4), and the existence of the carbon framework is demonstrated by SEM-energy dispersive X-ray (SEM-EDX) (Supplementary Fig. S5) and Raman spectra (D band and G band of carbon materials are observed in Figure 2i). The differences in the mapping images of C, Sn and O further confirm the geometrical entrapment of $\mathrm{SnO}_{2} \mathrm{NPs}$ in carbon inverse opal. In Figure $2 j-1$, the mapping image of $\mathrm{C}$ element covers the entire inverse opal with a continuous distribution while the distributions of $\mathrm{Sn}$ and $\mathrm{O}$ elements are segregated into clusters. Actually, the carbon materials constitute the entire host so their signals cover the whole area of inverse opal while the $\mathrm{SnO}_{2}$ NPs are just entrapped in the carbon host, thus showing discrete distribution.

$\mathrm{Li}$ ion batteries performances of the $\mathrm{SnO}_{2} @ 3 \mathrm{DOM}$ electrode. The LIBs performances of the $\mathrm{SnO}_{2} @ 3 \mathrm{DOM}$ were investigated using coin-type half-cells with a $\mathrm{Li}$ counter electrode and reference electrode. The cyclic voltammogram (CV) curves of the $\mathrm{SnO}_{2} @$ 3DOM for the first 5 cycles are shown in Figure 3a. The CV curves from the 2 nd to 5 th cycle share similar shape but there are some differences between the 1st and subsequent cycles. As previously demonstrated $^{32,33}$, the electrochemical interaction between lithium and $\mathrm{SnO}_{2}$ includes two steps given by the reactions: $\mathrm{SnO}_{2}+4 \mathrm{Li}^{+}$ $+4 \mathrm{e}^{-} \rightarrow \mathrm{Sn}+2 \mathrm{Li}_{2} \mathrm{O}(1) ; \mathrm{Sn}+x \mathrm{Li}^{+}+x \mathrm{e}^{-} \leftrightarrow \mathrm{Li}_{x} \mathrm{Sn}(0<x \leq 4.4)$ (2). In the first $\mathrm{CV}$ curve, the reduction peak at 0.95 should be attributed to the formation of a solid electrolyte interface (SEI) layer and $\mathrm{Li}_{2} \mathrm{O}$. The sharp reduction peaks below $0.5 \mathrm{~V}$ during subsequent discharging and the oxidation peaks at $0.51 \mathrm{~V}$ and $1.27 \mathrm{~V}$ during charging correspond to the reversible formation of $\mathrm{Li}_{x} \mathrm{Sn}$ alloys ${ }^{33,34}$. Figure 3b shows the cycling stability of $\mathrm{SnO}_{2} @ 3 \mathrm{DOM}$ for 500 discharge/charge cycles at a current density of $0.5 \mathrm{~A} \mathrm{~g}^{-1}$. In the 1st cycle, the discharge and charge capacities of $\mathrm{SnO}_{2} @ 3 \mathrm{DOM}$ are 1659.0 and $825.5 \mathrm{mAh}^{-1}$, respectively, corresponding to a coulombic efficiency of $49.76 \%$. The irreversible capacity should be mainly caused by the formation of SEI films on porous framework of the electrode although this could be improved by pre-lithiation in future optimization. In the 2 nd cycle, the corresponding coulombic efficiency quickly increases from $49.76 \%$ to $91.95 \%$, and the $\mathrm{SnO}_{2} @$ 3DOM delivers a high reversible capacity of $759.6 \mathrm{mAh}^{-1}$. In the 500 th cycle, a high reversible capacity of $715.2 \mathrm{mAh} \mathrm{g}^{-1}$ is still achieved, which retains $94.15 \%$ reversible capacity of the 2 nd cycle, accounting for $91.57 \%$ of the theoretical capacity of $\mathrm{SnO}_{2}$. Apparently, the $\mathrm{SnO}_{2} @ 3 \mathrm{DOM}$ presents excellent cycling stability without any significant capacity loss at a current density of $0.5 \mathrm{~A}$ $\mathrm{g}^{-1}$. These results are superior to those reported for $\mathrm{SnO}_{2}$ 


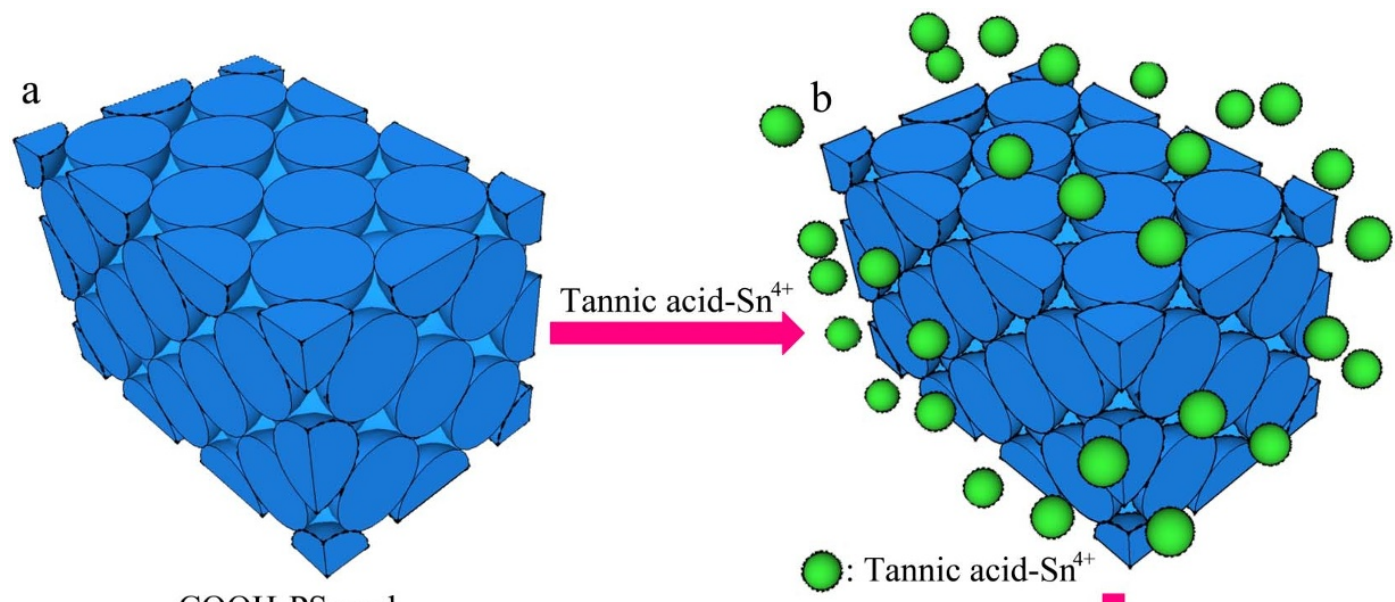

COOH-PS opal

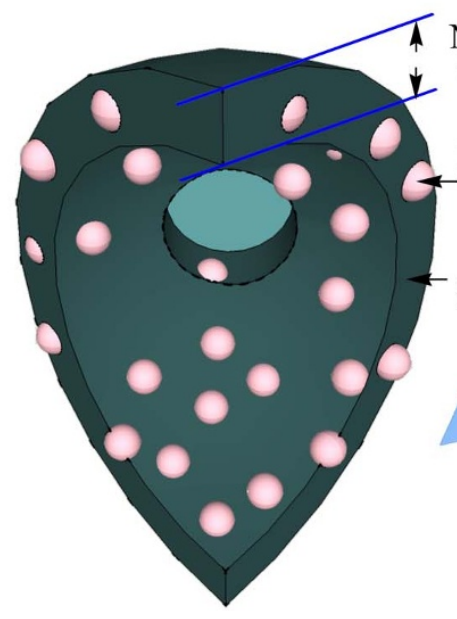

Nanometer thick wall

Entrapped

$\mathrm{SnO} 2 \mathrm{NPs}$

Carbon

inverse opal

Tannic acid-Sn

Vaccum

annealing
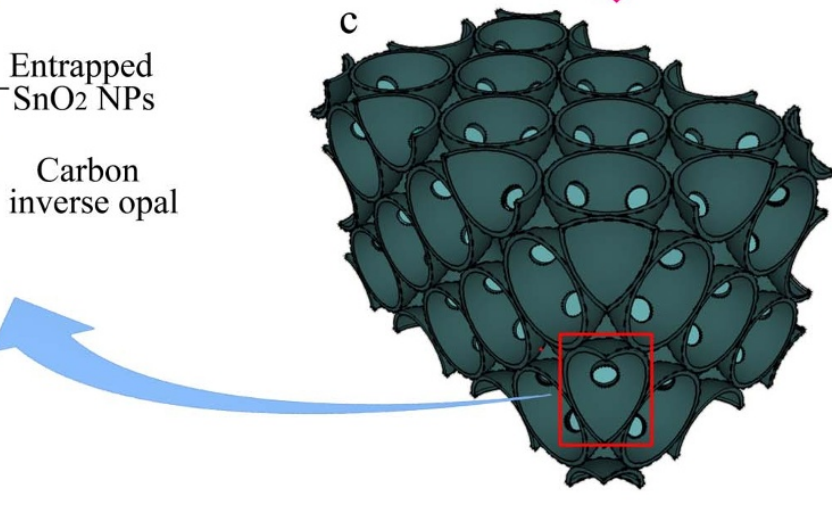

$\mathrm{SnO} 2 @ 3 \mathrm{DOM}$

Figure 1 | Schematic illustration showing the preparation of $\mathrm{SnO}_{2} @ 3 \mathrm{DOM}$ electrode.

encapsulated in carbon shell ${ }^{35}$, hybrid with carbon nanotube ${ }^{36}$ or graphene ${ }^{37}$. The cycling performance of 3DOM carbon inverse opal at a current density of $0.5 \mathrm{~A} \mathrm{~g}^{-1}$ is also conducted while its reversible capacity during cycling is very limited (Supplementary Fig. S6), thus suggesting that the high reversible capacity of $\mathrm{SnO}_{2} @$ 3DOM should be mainly derived from the $\mathrm{SnO}_{2}$ nanoparticles. Compared with the electrodes that are prepared by direct coating $\mathrm{SnO}_{2}$ on $3 \mathrm{DOM}$ carbon inverse opal $\left(\sim 200 \mathrm{mAh} \mathrm{g}^{-1}\right.$ in the 30 th cycle), the $\mathrm{SnO}_{2} @ 3 \mathrm{DOM}$ still exhibits superior advantages both in capacity and cycling stability ${ }^{24}$.

In general, poor charge transfer kinetics will lead to an increase in the impedance of batteries ${ }^{38,39}$. To confirm that the entrapment of $\mathrm{SnO}_{2} \mathrm{NPs}$ in carbon inverse opal can be beneficial for charge transfer kinetics, we tested the electrochemical impedance spectroscopy (Figure 3c) of the $\mathrm{SnO}_{2} @ 3 \mathrm{DOM}$ after 3 and 500 cycles at a current density of $0.5 \mathrm{~A} \mathrm{~g}^{-1}$, and an equivalent circuit was used for fitting the electrochemical impedance spectra. As shown in Figure $3 c$, the intercept at the $\mathrm{Z}^{\prime}$ axis at high frequency corresponds to the ohmic resistance $\left(R_{\Omega}\right)$, which represents the total resistance of the electrolyte, separator and electrical contacts. The semicircle in the middle frequency range indicates the charge transfer resistance (Rct), and the inclined line at lower frequency represents the Warburg impedance (W). Based on the model fitting, the charge transfer resistance (Rct) is $\sim 62 \Omega$ after 3 cycles, which demonstrates the fast charge transfer kinetics in batteries. More interesting, the charge transfer resistance is further decreased to $\sim 32 \Omega$ after 500 cycles, corresponding to improved charge transfer kinetics. Such phenomenon is also evident for the batteries based on CoO@3DOM electrode (see below in Figure $5 \mathrm{c}$ ). These surprising results are due to the unique structure of the designed electrodes. Usually, the active materials will pulverize and peel off from the electrode at high current density, thus leading to significantly increased impedance. However, in $\mathrm{SnO}_{2} @ 3 \mathrm{DOM}$, it is impossible for the $\mathrm{SnO}_{2}$ NPs to substantially peel off from electrode because they are geometrically confined within the framework of the carbon inverse opal. Based on TEM analysis, the $\mathrm{SnO}_{2}$ nanoparticles are still well entrapped in the carbon framework without serious aggregation even after 500 discharge/charge cycles (Supplementary Fig. S7), thus confirming that the 3DOM carbon framework indeed provides effective stabilization to the $\mathrm{SnO}_{2}$ nanoparticles. Although the pulverization of $\mathrm{SnO}_{2} \mathrm{NPs}$ is inevitable during cycling, the electronic conductivity of the pulverized $\mathrm{SnO}_{2} \mathrm{NPs}$ with the carbon host can still be re-established during cycling, and therefore improved charge transfer kinetics with lower impedance can be exhibited. To further demonstrate this proposed mechanism, deep discharge/ charge cycling of $\mathrm{SnO}_{2} @ 3 \mathrm{DOM}$ is carried out at even higher current density of $1.0 \mathrm{~A} \mathrm{~g}^{-1}$ for 500 cycles, where the cracking and pulverization of $\mathrm{SnO}_{2}$ will be more severe in the initial cycles. As shown in Figure 3b, the discharge capacity of $\mathrm{SnO}_{2} @ 3 \mathrm{DOM}$ at a current density of $1.0 \mathrm{~A} \mathrm{~g}^{-1}$ initially exhibits a relatively more obvious decrease in the first dozens of cycles, but then steadily increases back in subsequent cycles, and gradually reaches equilibrium around the 470th cycle. Based on the proposed mechanism, these cycling behaviors are 
a

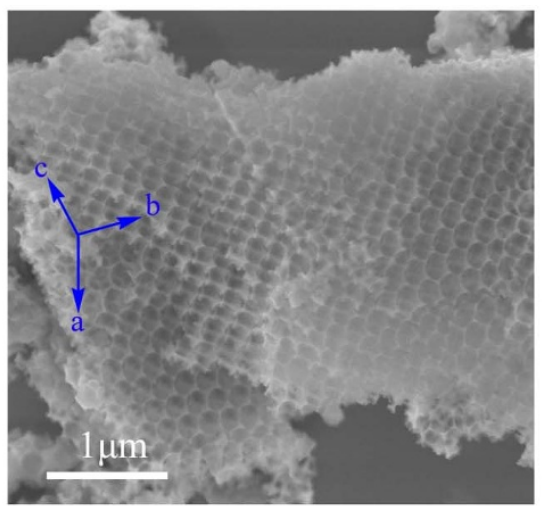

$\mathrm{d}$

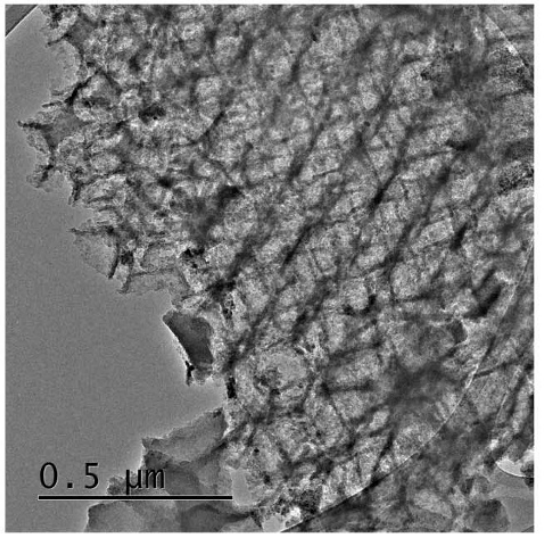

g

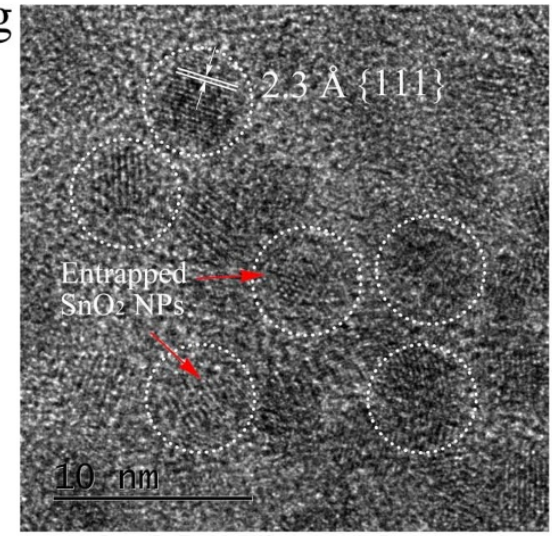

$\mathrm{j}$

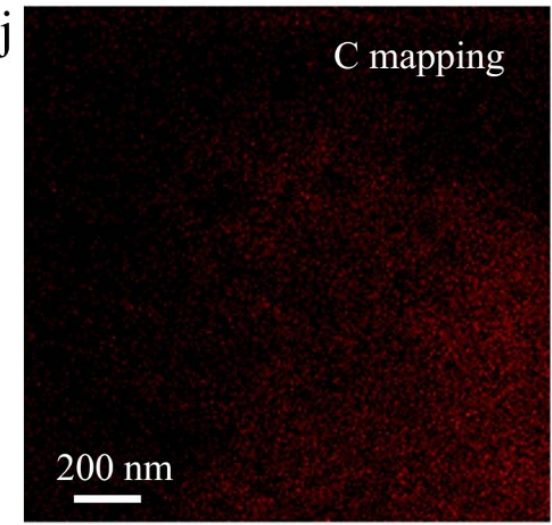

b

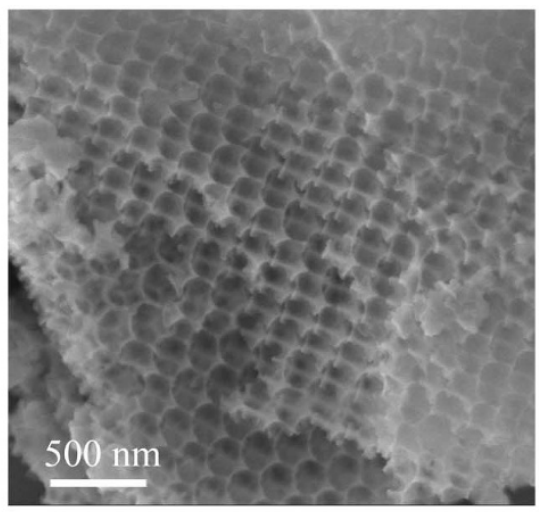

e

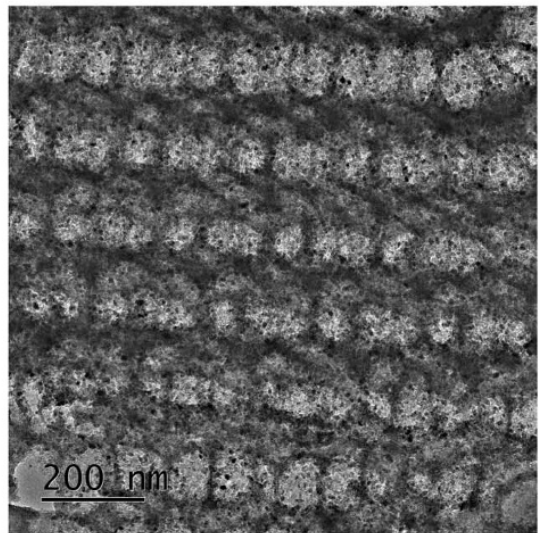

$\mathrm{h}$

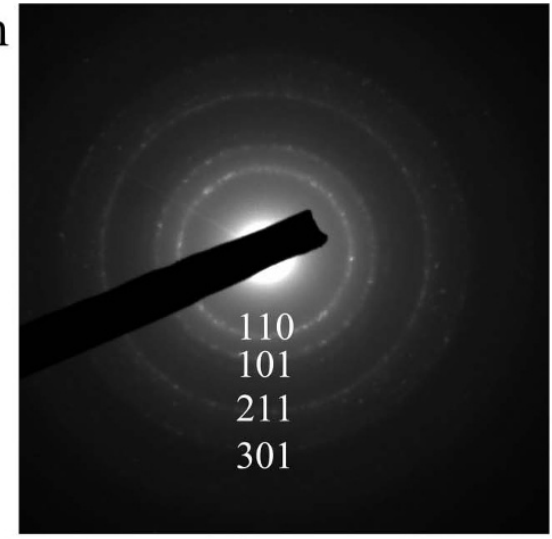

$\mathrm{k}$

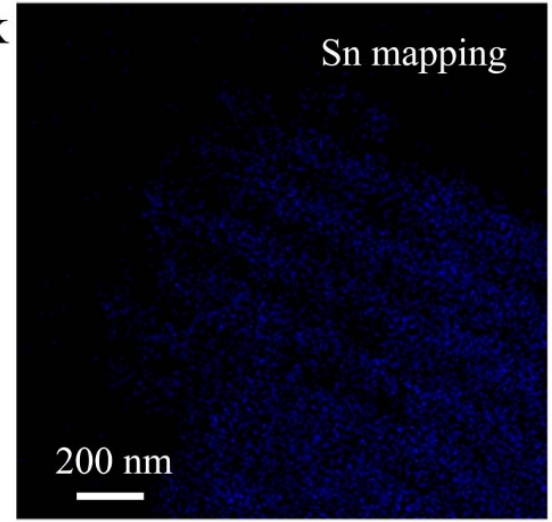

c

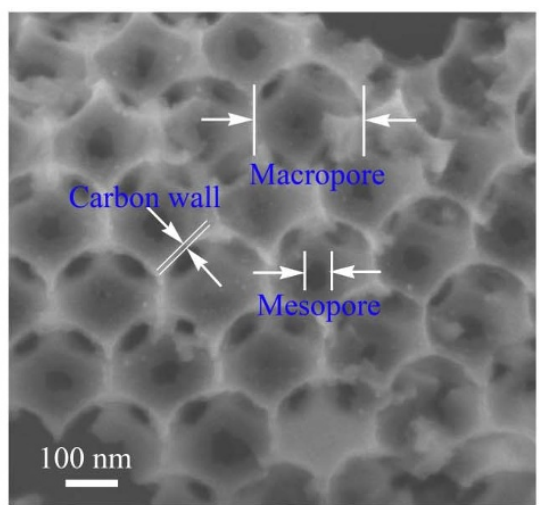

f

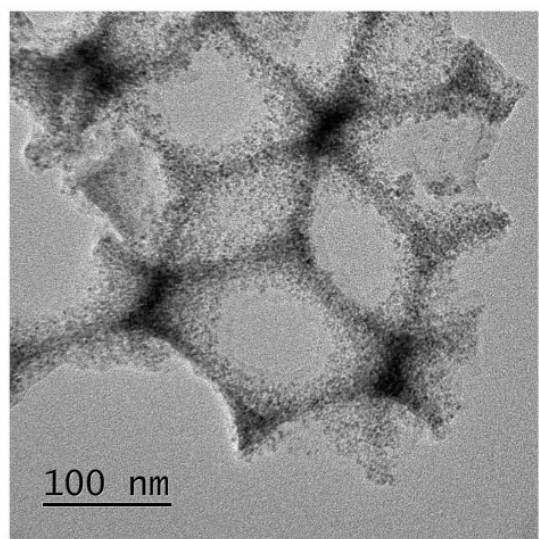

i

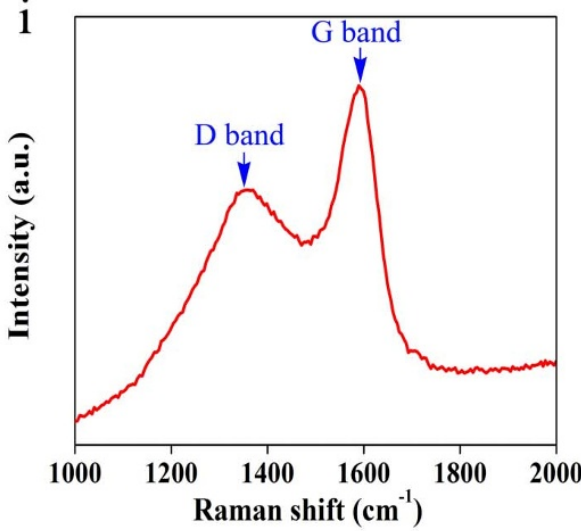

1

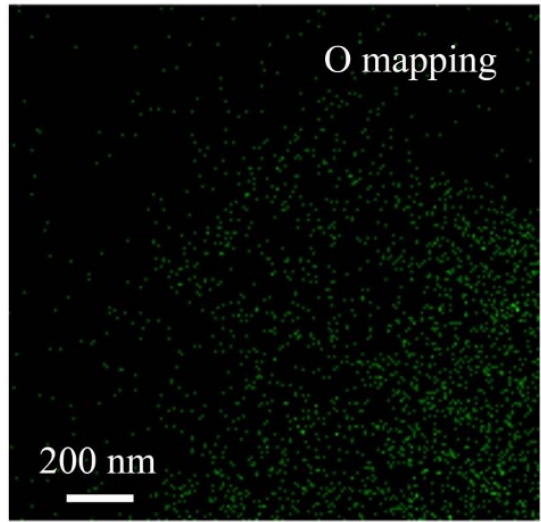

Figure 2| Microstructure of $\mathrm{SnO}_{2} @ 3 D O M$ electrode. 

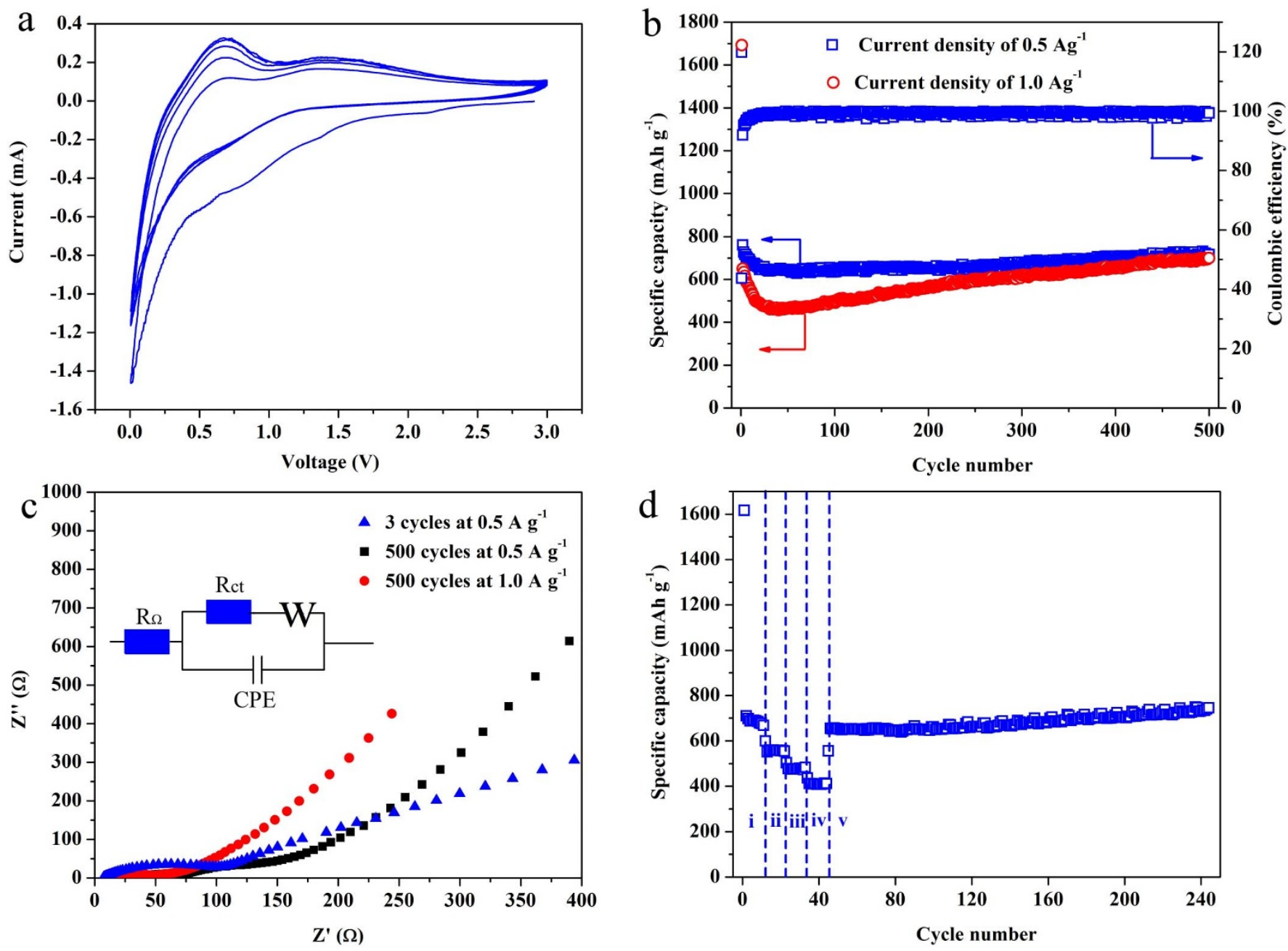

Figure 3 Li-ion battery performances of $\mathrm{SnO}_{2} @ 3 \mathrm{DOM}$.

reasonable and explainable. Compared with the cycling performances at $0.5 \mathrm{~A} \mathrm{~g}^{-1}$, the more obvious capacity decrease at the initial stage is caused by the more severe pulverization of $\mathrm{SnO}_{2}$ at the doubled current density $\left(1.0 \mathrm{~A} \mathrm{~g}^{-1}\right)$. After that, the electronic conductivity of the pulverized particles with the carbon host is gradually reestablishing, and accordingly, the battery exhibits slow but steadily increased capacities with further cycling. Upon stable re-establishment of the electronic conductivity, the cycling performance of the $\mathrm{SnO}_{2} @$ $3 \mathrm{DOM}$ reaches equilibrium. Compared with the initial several cycles, the substantial decrease in impedance at $1.0 \mathrm{~A} \mathrm{~g}^{-1}$ after 500 cycles confirms the re-establishment of good charge-transfer (Figure 3c). Notably, the $\mathrm{SnO}_{2} @ 3 \mathrm{DOM}$ delivers a high capacity of $\sim 700 \mathrm{mAh}$ $\mathrm{g}^{-1}$ in the 500th cycle at current density of $1.0 \mathrm{~A} \mathrm{~g} \mathrm{~g}^{-1}$. To the best of our knowledge, this is the longest reported lifespan under such high current density for $\mathrm{SnO}_{2}$-based anode materials used in LIBs.

Rate capability is another important criterion for high-performance LIBs. In Figure 3d, the rate capabilities of $\mathrm{SnO}_{2} @ 3 \mathrm{DOM}$ were tested at current densities of $0.5,1.0,1.5$ and $2.0 \mathrm{~A} \mathrm{~g}^{-1}$, and the corresponding discharge capacities in the 10th cycle are 676.5, 556.7, 473.5 and $414.2 \mathrm{mAh} \mathrm{g}^{-1}$, respectively. Notably, the discharge capacity of $\mathrm{SnO}_{2} @ 3 \mathrm{DOM}$ could immediately increase back without any delay when the current density decreased from 2.0 to $0.5 \mathrm{~A} \mathrm{~g}^{-1}$. The discharge capability maintains at $745.7 \mathrm{mAh} \mathrm{g}^{-1}$ in the 100th cycle, which shows that the well designed $\mathrm{SnO}_{2} @ 3 \mathrm{DOM}$ can be operated under such current densities with pretty good rate capabilities. To further gain a measurement of the rate capability under conditions where fast ion and electron transport and structural stability of active materials should be essential, the discharge capability of
$\mathrm{SnO}_{2} @ 3 \mathrm{DOM}$ is tested at different high current densities. As shown in Supplementary Fig. S8, the $\mathrm{SnO}_{2} @ 3 D O M$ delivers discharge capacities of 445.4, 368.2, 321.0, 285.8, 261.7, 216.0 and $147.5 \mathrm{mAh} \mathrm{g}^{-1}$ in the 50th cycle at the current densities of 1.0, 2.0, 3.0, 4.0, 5.0, 8.0 and $10.0 \mathrm{~A} \mathrm{~g}^{-1}$, respectively. Upon returning the current density from 10.0 to $1.0 \mathrm{~A} \mathrm{~g}^{-1}$, the discharge capacity can gradually increase back to $\sim 600 \mathrm{mAh} \mathrm{g}^{-1}$ although there is some delay in capacity recovery. The significance of the above results strongly demonstrates that high-performance electrodes with outstanding stability and rate capability are possible by entrapping electrode active nanomaterials in macroporous carbon inverse opal.

Synthesis and characterization of the CoO@3DOM electrode. Our strategy can be extended to synthesize other 3DOM electrodes because plant tannins have an outstanding chelating ability towards various transition metal ions ${ }^{40-43}$. To verify this, we prepared CoO@3DOM electrode by the same strategy as illustrated in Figure 1. XRD analysis demonstrates the presence of $\mathrm{CoO}$ in CoO@3DOM (Supplementary Fig. S9), and the analysis of Raman spectroscopy confirms the formation of carbon framework (Supplementary Fig. S10). The morphology of periodic porosities, interconnected macropores on the order of $220 \mathrm{~nm}$, and smaller mesopores on the order of $40-50 \mathrm{~nm}$, are also evident in FE-SEM images shown in Figure $4 \mathrm{a}-\mathrm{c}$. The periodic porosity of the 3DOM electrode is clearly observed in Figure $4 \mathrm{~d}$, and the CoO NPs with diameter of $5 \sim 10 \mathrm{~nm}$ are well entrapped in the 3DOM framework, as shown in Figure 4e. In Figure 4f, the CoO NPs with clear lattice fringes are confined in the carbon matrices without serious 
a

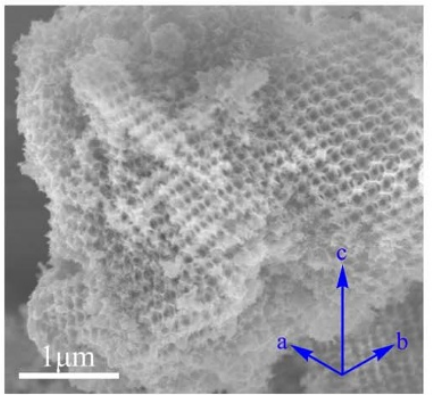

d
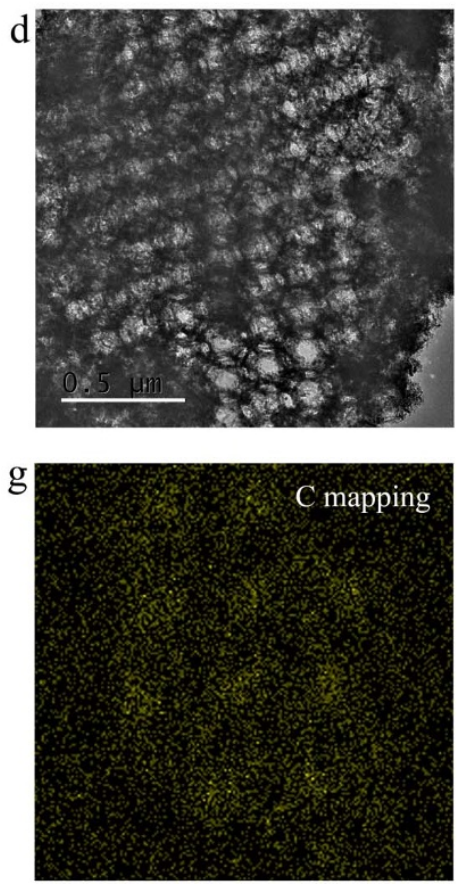

b
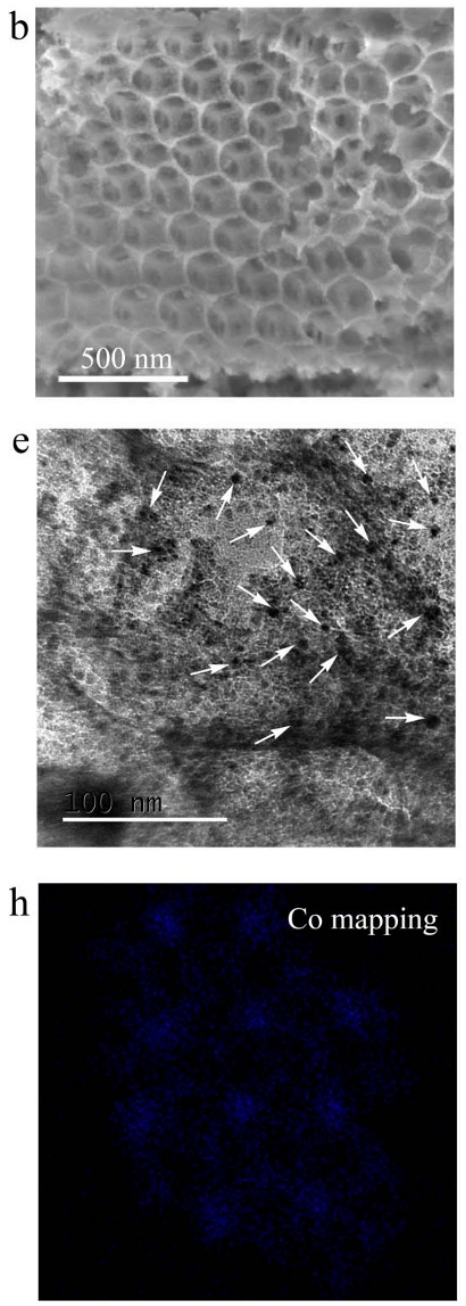
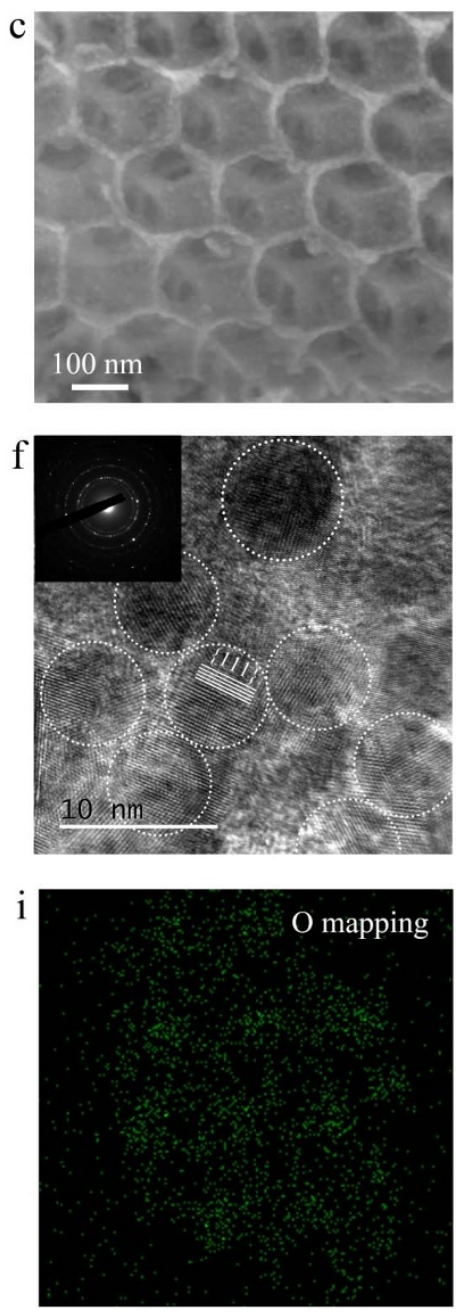

Figure $4 \mid$ Microstructure of CoO@3DOM.

aggregation, and the SAED analysis reveals their crystalline nature (inset in Figure 4f). Further EDX elemental mapping analyses confirm that the CoO NPs are successfully entrapped within the carbon inverse opal (Figure 4g-i). Moreover, the CoO@3DOM still exhibits low charge transfer resistance (Rct) of $97 \Omega$ (Figure $5 \mathrm{c}$ ), thus revealing fast $\mathrm{Li}^{+}$diffusion and electron transfer kinetics.

Li-ion batteries performances of the CoO@3DOM electrode. The discharge/charge cycling performance of CoO@3DOM is first conducted at a current density of $0.5 \mathrm{~A} \mathrm{~g}^{-1}$ in the voltage range of 0.005-3.0 V. Figure 5a shows the galvanostatic discharge and charge voltage profiles generated by the CoO@3DOM in the 1st, 2nd and 500th cycles. In the 1st cycle, the discharge and charge of theCoO@ 3DOM occurs at 1375.5 and $736.7 \mathrm{mAh} \mathrm{g}^{-1}$, respectively, and the coulombic efficiency is around $53.56 \%$. In the 2nd cycle, the CoO@ 3DOM exhibits a reversible capacity of $718.6 \mathrm{mAh} \mathrm{g}^{-1}$, and its coulombic efficiency is quickly increased to $94.58 \%$. Notably, the discharge voltage profiles of the 500th and the 2nd cycles almost overlap with each other, suggesting a high cycling stability. Figure 5b shows the detailed cycling performances of CoO@ 3DOM. At a current density of $0.5 \mathrm{~A} \mathrm{~g}^{-1}$, the discharge capacity just slightly decreases in the initial dozens of cycles and then steadily increases back, maintaining a high level of discharge capacity. In the 500th cycle, it delivers a capacity of $682.3 \mathrm{mAh}$ $\mathrm{g}^{-1}$, accounting for $95.43 \%$ of the theoretical capacity. At a current density of $2.0 \mathrm{~A} \mathrm{~g}^{-1}$, the CoO@3DOM shows a more obvious capacity decrease in the initial cycles due to the more severe pulverization of $\mathrm{CoO}$ at such high current density, and subsequently, the capacity gradually increase back and reaches an equilibration around the 400th cycle. In the 1000th cycle, CoO@3DOM can still deliver a high capacity of $673.7 \mathrm{mAh} \mathrm{g}^{-1}$, accounting for $94.17 \%$ of the theoretical capacity. Hence, the CoO@3DOM also exhibits an excellent long-term cycling stability. Compared with the initial stage of cycling, the impedance of batteries substantially decreased from 97 to $28 \Omega$ when cycled from the 3 rd to 500 th cycle at a current density of $0.5 \mathrm{~A} \mathrm{~g}^{-1}$, as shown in Figure $5 \mathrm{c}$. These changes of impedance are similar to those of batteries based on the $\mathrm{SnO}_{2} @$ 3DOM electrode, revealing the re-establishment of good charge transfer during discharge/charge cycling. According to the literature ${ }^{44,45}$, other previous researches also reported the capacities of electrodes increase gradually after long term cycles, and they considered this might result from the improved Li-ion diffusion kinetics by an activation and stabilization process during cycling. Figure 5d shows the rate performance of the CoO@3DOM electrode at 1.0-10.0 $\mathrm{A} \mathrm{g}^{-1}$. The discharge capacities of the $\mathrm{CoO} @$ 3DOM are 560.8, 479.9, 351.4, 297.8, 265.2, 238.9 and $200.6 \mathrm{mAh} \mathrm{g}^{-1}$, respectively, when performed 50 cycles at 1.0, 2.0, 3.0, 4.0, 5.0, 8.0A and $10.0 \mathrm{~A} \mathrm{~g} \mathrm{~g}^{-1}$, respectively, and can gradually increase back to $\sim 590 \mathrm{mAh} \mathrm{g}^{-1}$ at $1.0 \mathrm{~A} \mathrm{~g}^{-1}$. If the rate performance is conducted at relatively low current density $\left(0.5-2.0 \mathrm{~A} \mathrm{~g}^{-1}\right)$, the capacity recovery will be faster when returning the discharge rate from a higher current density to a lower one (Supplementary Fig. S11). Again, the good electrochemical performances of the CoO@3DOM at high current densities demonstrate the feasibility of our strategy. 

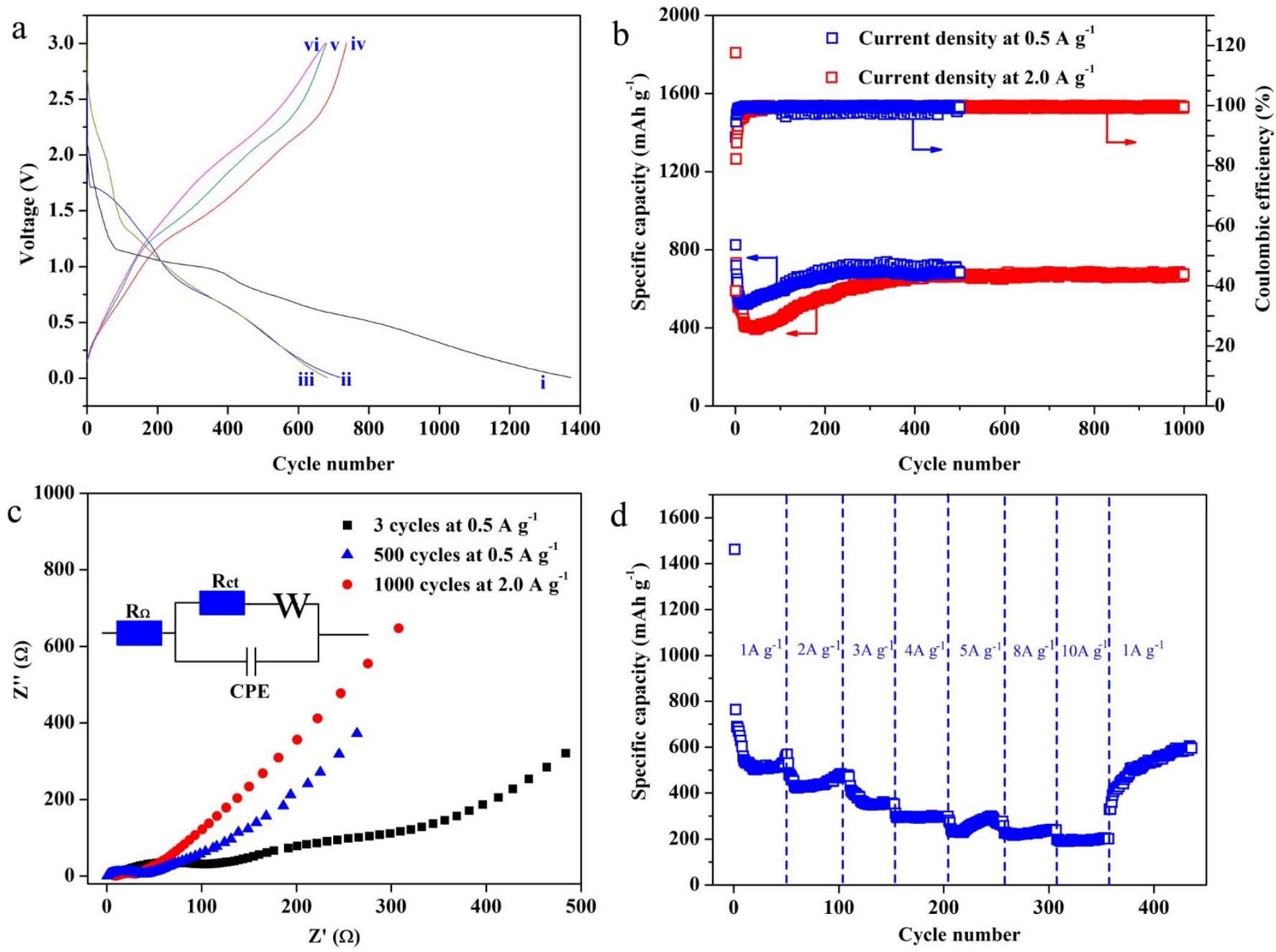

Figure 5 | Li-ion battery performances and electrochemical impedance spectroscopy of CoO@3DOM.

\section{Discussion}

In summary, we have demonstrated a versatile concept for the synthesis of three-dimensional ordered macroporous conductive electrode that displays a continuous and efficient pathway for fast ion and electron transport as well as stably geometrical constrains to active materials. This concept is established based on the integration of ordered macroporous carbon inverse opal with entrapped active nanoparticles. The carbon inverse opal not only serves as the interpenetrating continuous conductive network but also acts as a dimensional constraint to the active nanoparticles. The entrapment of the active nanoparticles provides intimate contact with the carbon inverse opal and also completely avoids the detachment problem of the active materials from the electrode, thus simultaneously affording fast ion and electron transport as well as superior cycling stability. These are vital to the success of all high-performance anodes to ensure large reversible capacity at high current densities. As demonstrated, the as-prepared electrode can deliver up to $94.17 \%$ of theoretical capacity over 1000 discharge/charge cycles, and exhibit excellent rate capability at the current density range of 1.0-10.0 A $\mathrm{g}^{-1}$. It also should be noted that porous structured electrodes often suffer from relatively low volumetric energy density, and our 3D anode materials also have such problem because of their periodic macroporous structure. To elevate such problem, further improvements are still required. Owing to the flexibility of our concept, it is possible to fabricate a class of new high-performance electrodes previously considered not feasible due to their low electronic and ionic conductivity, and we will report these researches in the forthcoming papers.

\section{Methods}

Opal template fabrication. The synthesis of carboxylic polystyrene (COOH-PS) spheres was fabricated as described previously ${ }^{46,47}$. Typically, $100 \mathrm{ml}$ of deionized water, $0.12 \mathrm{~g}$ of $\mathrm{NaHCO}_{3}, 5 \mathrm{ml}$ of styrene and $0.5 \mathrm{ml}$ of acrylic acid were added into a three-necked flask equipped with a reflux condenser and a mechanical stirrer where potassium persulfate served as the initiator. The reaction was carried out under a nitrogen atmosphere at $70^{\circ} \mathrm{C}$ for $6 \mathrm{~h}$, followed by reacting at $90^{\circ} \mathrm{C}$ for $1 \mathrm{~h}$. The resulting product was purified by centrifugation/dispersion cycles in water, and further aggregated to form self-assembled COOH-PS opal by high speed centrifugation.

$\mathrm{SnO}_{2} @ 3 \mathrm{DOM}$ fabrication. $0.02 \mathrm{~g}$ of tannic acid and $0.104 \mathrm{~g}$ of $\mathrm{SnCl}_{4} \cdot x \mathrm{H}_{2} \mathrm{O}$ were mixed in $1.0 \mathrm{ml}$ of deionized water. $0.05 \mathrm{~g}$ of $\mathrm{COOH}-\mathrm{PS}$ opal was gently added to the above solution, followed by vacuum drying and thermal annealing in vacuum at $400^{\circ} \mathrm{C}$ for $2.0 \mathrm{~h}$.

CoO@3DOM fabrication. $0.02 \mathrm{~g}$ of tannic acid and $0.291 \mathrm{~g}$ of $\mathrm{Co}\left(\mathrm{NO}_{3}\right)_{2} \bullet 6 \mathrm{H}_{2} \mathrm{O}$ were mixed in $1.0 \mathrm{ml}$ of deionized water. $0.05 \mathrm{~g}$ of $\mathrm{COOH}-\mathrm{PS}$ opal was gently added to the above solution, followed by vacuum drying and thermal annealing in vacuum at $400^{\circ} \mathrm{C}$ for $2.0 \mathrm{~h}$.

3DOM carbon inverse opal. $0.02 \mathrm{~g}$ of tannic acid was mixed in $1.0 \mathrm{ml}$ of deionized water. $0.05 \mathrm{~g}$ of $\mathrm{COOH}-\mathrm{PS}$ opal was gently added to the above solution, followed by vacuum drying and thermal annealing in vacuum at $400^{\circ} \mathrm{C}$ for $2.0 \mathrm{~h}$.

Electrochemical measurement. Negative electrodes were comprised $70 \mathrm{wt} \%$ of $\mathrm{SnO}_{2} @ 3 \mathrm{DOM}$ (or $\mathrm{CoO} @ 3 \mathrm{DOM}$ ), 20 wt\% carbon nanotubes, and 10 wt $\%$ poly(vinylidene fluoride) binder. The anode materials were slurry-cast from $\mathrm{N}$ - 
methyl-2-pyrrolidinone onto a $\mathrm{Cu}$ foil, and vacuum-dried at $50^{\circ} \mathrm{C}$ to completely remove the solvent. The electrochemical properties of the obtained working electrodes were measured using two-electrode CR2032 (3 V) coin-type cells with lithium foil serving as both counter and reference electrodes under ambient temperature. The electrolyte was $1 \mathrm{M} \mathrm{LiPF}_{6}$ in a 50:50 (w/w) mixture of ethylene carbonate (EC) and dimethyl carbonate (DMC). Cell assembly was carried out in an argon-filled glove box with both moisture and oxygen contents below $1.0 \mathrm{ppm}$. Galvanostatic discharge/charge tests were performed using a NEWARE battery tester at a voltage window of $0.005-3.0 \mathrm{~V}$. The specific capacity of the anode is calculated based on the mass of $\mathrm{SnO}_{2} @ 3 \mathrm{DOM}$ (or $\mathrm{CoO} @ 3 \mathrm{DOM}$ ) contained in the anode composites. According to electrochemical tests, the discharge capacity contribution of carbon nanotubes to the $\mathrm{SnO}_{2} @ 3 \mathrm{DOM}$ (or CoO@3DOM) electrode is very limited (Supplementary Fig. S12).

Characterizations. Ultraviolet-visible (UV-vis) spectra analyses were conducted using UV-Vis spectrometer (Shimadzu UV-2501PC). Thermogravimetry analyses (TGA, Q500) were carried out in the temperature range $33-900^{\circ} \mathrm{C}$ at a heating rate of $15^{\circ} \mathrm{C} \mathrm{min}^{-1}$ in $\mathrm{N}_{2}$ flow. Wide angle X-ray diffraction patterns of the samples were recorded using a Bruker D8 Advance diffractometer using $\mathrm{Cu} \mathrm{K} \alpha$ radiation. Raman spectra and images were obtained by a WITeck CRM200 confocal microscopy Raman system with a piezocrystal controlled scanning stage. The morphology of the samples was characterized with a field emission scanning electron microscope (FESEM) system (JEOL, Model JSM-7600F) and a transmission electron microscope (TEM) system (JEOL, Model JEM-2010F) operating at $200 \mathrm{kV}$.

1. Tarascon, J. M. \& Armand, M. Issues and challenges facing rechargeable lithium batteries. Nature 414, 359-367 (2001).

2. Lee, S. W. et al. High-power lithium batteries from functionalized carbonnanotube electrodes. Nat. Nanotech. 5, 531-537 (2010).

3. Chan, C. K. et al. High-performance lithium battery anodes using silicon nanowires. Nat. Nanotech. 3, 31-35 (2008)

4. Kim, M. G. \& Cho, J. Reversible and high-capacity nanostructured electrode materials for Li-ion batteries. Adv. Funct. Mater. 19, 1497-1514 (2009).

5. Zhang, H .G., Yu, X. D. \& Braun, P. V. Three-dimensional bicontinuous ultrafastcharge and -discharge bulk battery electrodes. Nat. Nanotech. 6, 277-281 (2011).

6. Liang, S. Z., Zhu, X. F., Lian, P. C., Yang, W. S. \& Wang, H. H. Superior cycleperformance of Sn@C/graphene nanocomposite as an anode material for lithium-ion batteries. J. Solid State Chem. 184, 1400-1404 (2011).

7. Arico, A. S., Bruce, P., Scrosati, B., Tarascon, J. M. \& Van Schalkwijk, W Nanostructured materials for advanced energy conversion and storage devices. Nat. Mater. 4, 366-377 (2005).

8. Wu, Z. S. et al. Graphene anchored with $\mathrm{Co}_{3} \mathrm{O}_{4}$ nanoparticles as anode of lithium ion batteries with enhanced reversible capacity and cyclic performance. ACS nano 4, 3187-3194 (2010)

9. Yang, S. B. et al. Porous iron oxide ribbons grown on graphene for highperformance lithium storage. Sci. Rep. 2, (2012).

10. Poizot, P., Laruelle, S., Grugeon, S., Dupont, L. \& Tarascon, J. M. Nano-sized transition-metaloxides as negative-electrode materials for lithium-ion batteries. Nature 407, 496-499 (2000).

11. Zhang, W. J. A review of the electrochemical performance of alloy anodes for lithium-ion batteries. J. Power Sources 196, 13-24 (2011).

12. Li, H. Q. \& Zhou, H. S. Enhancing the performances of Li-ion batteries by carboncoating: present and future. Chem. Commun. 48, 1201-1217 (2012).

13. Qiu, Y. C., Yan, K. Y. \& Yang, S. H. Ultrafine tin nanocrystallites encapsulated in mesoporous carbon nanowires: scalable synthesis and excellent electrochemical properties for rechargeable lithium ion batteries. Chem. Commun. 46, 8359-8361 (2010).

14. Chockla, A. M. et al. Silicon nanowire fabric as a lithium ion battery electrode material. J. Am. Chem. Soc. 133, 20914-20921 (2011)

15. Wang, N., Xu, J. X. \& Guan, L. X. Synthesis and enhanced photocatalytic activity of tin oxide nanoparticles coated on multi-walled carbon nanotube. Mater. Res. Bull. 46, 1372-1376 (2011).

16. Bonino, C. A. et al. Electrospun carbon-tin oxide composite nanofibers for use as lithium ion battery anodes. ACS Appl. Mater. Interfaces 3, 2534-2542 (2011).

17. Zhu, X. J., Zhu, Y. W., Murali, S., Stollers, M. D. \& Ruoff, R. S. Nanostructured reduced graphene Oxide/ $\mathrm{Fe}_{2} \mathrm{O}_{3}$ composite as a high-performance anode material for lithium ion batteries. ACS Nano 5, 3333-3338 (2011).

18. Luo, B. et al. Graphene-confined Sn nanosheets with enhanced lithium storage capability. Adv. Mater. 24, 3538-3543 (2012).

19. Amin, R., Balaya, P. \& Maier, J. Anisotropy of electronic and ionic transport in $\mathrm{LiFePO}_{4}$ single crystals. Electrochem. Solid-State Lett. 10, A13-A16 (2007).

20. Lytle, J. C., Yan, H. W., Ergang, N. S., Smyrl, W. H. \& Stein, A. Structural and electrochemical properties of three-dimensionally ordered macroporous tin(IV) oxide films. J. Mater. Chem. 14, 1616-1622 (2004).

21. Timothy, S. et al. Three-dimensional electrodes and battery architectures. MRS Bull. 36, 523-531 (2011).

22. Sorensen, E. M. et al. Three-dimensionally ordered macroporous $\mathrm{Li}_{4} \mathrm{Ti}_{5} \mathrm{O}_{12}$ : effect of wall structure on electrochemical properties. Chem. Mater. 18, 482-489 (2006).

23. Kavan, L., Zukalova, M. T., Kalbac, M. \& Graetzel, M. Lithium insertion into anatase inverse opal. J. Electrochem. Soc. 151, A1301-A1307 (2004).
24. Lee, K. T., Lytle, J. C., Ergang, N. S., Oh, S. M. \& Stein, A. Synthesis and rate performance of monolithic macroporous carbon electrodes for lithium-ion secondary batteries. Adv. Funct. Mater. 15, 547-556 (2005).

25. Esmanski, A. \& Ozin, G. A. Silicon inverse-opal-based macroporous materials as negative electrodes for lithium ion batteries. Adv. Funct. Mater. 19, 1999-2010 (2009).

26. Landon, P. B., Gilleland, C. L. \& Glosser, R. Properties of metallo-dielectric opals and metallic inverse opal photonic crystals. J. Mater. Sci. : Mater. Electron. 18, S469-S472 (2007).

27. Orilall, M. C., Abrams, N. M., Lee, J., DiSalvo, F. J. \& Wiesner, U. Highly crystalline inverse opal transition metal oxides via a combined assembly of soft and hard chemistries. J. Am. Chem. Soc. 130, 8882-8883 (2008).

28. Guan, G. Q. et al. Preferential CO oxidation over catalysts with well-defined inverse opal structure in microchannels. Int. J. Hydrogen Energy 33, 797-801(2008).

29. Vu, A., Qian, Y. Q. \& Stein, A. Porous electrode materials for lithium-ion batteries - how to prepare them and what makes them special. Adv. Energy Mater. 2, 1056-1085 (2012).

30. Huang, J. H., Liu, Y. F. \& Wang, X. G. Selective adsorption of tannin from flavonoids by organically modified attapulgite clay. J. Hazard. Mater. 160, 382-387 (2008)

31. Lin, D. H. \& Xing, B. S. Tannic acid adsorption and its role for stabilizing carbon nanotube suspensions. Environ. Sci. Technol. 42, 5917-5923 (2008).

32. Lou, X. W., Li, C. M. \& Archer, L. A. Designed synthesis of coaxial SnO2@carbon hollow nanospheres for highly reversible lithium storage. Adv. Mater. 21 , 2536-2539 (2009).

33. Zhu, X. J., Zhu, Y. W., Murali, S., Stoller, M. D. \& Ruoff, R. S. Reduced graphene oxide/tin oxide composite as an enhanced anode material for lithium ion batteries prepared by homogenous coprecipitation. J. Power Sources 196, 6473-6477 (2011).

34. Yin, X. M. et al. One-step synthesis of hierarchical $\mathrm{SnO}_{2}$ hollow nanostructures via self-assembly for high power lithium ion batteries. J. Phys. Chem. C 114, 8084-8088 (2010).

35. Lee, K. T., Jung, Y. S. \& Oh, S. M. Synthesis of tin-encapsulated spherical hollow carbon for anode material in lithium secondary batteries. J. Am. Chem. Soc. 125, 5652-5653 (2003).

36. Fu, Y. B., Ma, R. B., Shu, Y., Cao, Z. \& Ma, X. H. Preparation and characterization of $\mathrm{SnO}_{2}$ /carbon nanotube composite for lithium ion battery applications. Mater. Lett. 63, 1946-1948 (2009).

37. Li, Y. M., Lv, X. J., Lu, J. \& Li, J. H. Preparation of $\mathrm{SnO}_{2}$-nanocrystal/graphenenanosheets composites and their lithium storage ability. J. Phys. Chem. C 114, 21770-21774 (2010).

38. Szczech, J. R. \& Jin, S. Nanostructured silicon for high capacity lithium battery anodes. Energy Environ. Sci. 4, 56-72 (2011).

39. Wu, H. et al. Aligned $\mathrm{NiO}$ nanoflake arrays grown on copper as high capacity lithium-ion battery anodes. J. Mater. Chem. 22, 19821-19825 (2012).

40. Quideau, S., Deffieux, D., Douat-Casassus, C. \& Pouysegu, L. Plant polyphenols: chemical properties, biological activities, and synthesis. Angew. Chem. Int. Ed. 50, 586-621 (2011)

41. Ma, H. W., Liao, X. P., Liu, X. \& Shi, B. Recovery of platinum(IV) and palladium(II) by bayberry tannin immobilized collagen fiber membrane from water solution. J. Membr. Sci. 278, 373-380 (2006).

42. Mao, H., Ma, J., Liao, Y., Zhao, S. L. \& Liao, X. P. Using plant tannin as natural amphiphilic stabilizer to construct an aqueous-organic biphasic system for highly active and selective hydrogenation of quinoline. Catal. Sci. Technol. 3, 1612-1617 (2013).

43. Nakano, Y., Takeshita, K. \& Tsutsumi, T. Adsorption mechanism of hexavalent chromium by redox within condensed-tannin gel. Water Res. 35, 496-500 (2001)

44. Zeng, L. X., Zheng, C., Xia, L. C., Wang, Y. X. \& Wei, M. D. Ordered mesoporous $\mathrm{TiO}_{2}-\mathrm{C}$ nanocomposite as an anode material for long-term performance lithiumion batteries. J. Mater. Chem. A 1, 4293-4299 (2013).

45. Zeng, L. X. et al. $\mathrm{ZnV}_{2} \mathrm{O}_{4}$-CMK nanocomposite as an anode material for rechargeable lithiumion batteries. J. Mater. Chem. 22, 14284-14288 (2012).

46. Lu, Z. Y. et al. Monodisperse magnetizable silica composite particles from heteroaggregate of carboxylic polystyrene latex and $\mathrm{Fe}_{3} \mathrm{O}_{4}$ nanoparticles. Nanotechnology 19, 055602 (2008).

47. Wang, P. H. \& Pan, C. Y. Preparation of styrene/acrylic acid copolymer microspheres: polymerization mechanism and carboxyl group distribution. Colloid Polym. Sci. 280, 152-159 (2002).

\section{Acknowledgements}

This work was supported by Singapore Ministry of Education (MOE2010-T2-1-017), A*STAR SERC grant 1021700144, NRF2009EWT-CERP001-026 (Singapore), Singapore National Research Foundation under CREATE program: EMobility in Megacities, and Singapore MPA 23/04.15.03 RDP 020/10/113 grant.

\section{Author contributions}

X. H. and H. H. H conceived the idea. X. H. and Z. L. carried out materials fabrication. X. H., J. C. and H. Y. performed materials characterizations and electrochemical tests. X. H., 
H. H. H. and Q. Y. drafted the manuscript. All authors analyzed and discussed the experimental results.

\section{Additional information}

Supplementary information accompanies this paper at http://www.nature.com/ scientificreports

Competing financial interests: The authors declare no competing financial interests.
How to cite this article: Huang, X. et al. Carbon inverse opal entrapped with electrode active nanoparticles as high-performance anode for lithium-ion batteries. Sci. Rep. 3, 2317; DOI:10.1038/srep02317 (2013)

(c) (i) (5) $\Theta$ This work is licensed under a Creative Commons Attribution-

NonCommercial-NoDerivs 3.0 Unported license. To view a copy of this license, visit http://creativecommons.org/licenses/by-nc-nd/3.0 